\title{
Toll-like receptor 4 /nuclear factor-kappa B pathway is involved in radicular pain by encouraging spinal microglia activation and inflammatory response in a rat model of lumbar disc herniation
}

\author{
Lirong Zhu ${ }^{1,2, *}$, Yangliang Huang ${ }^{3, *}$, Yuming $\mathrm{Hu}^{4}$, Qian Tang ${ }^{1,2}$, and Yi Zhong ${ }^{1,2}$ \\ 'Key Laboratory of Neuroscience, School of Basic Medical Science, Guangzhou Medical University, Guangzhou, China \\ 'Institute of Neuroscience and Department of Neurology, The Second Affiliated Hospital of Guangzhou Medical University, Guangzhou, China \\ ${ }^{3}$ Department of Spine Surgery, First Affiliated Hospital of Sun Yat-Sen University, Guangzhou, China \\ ${ }^{4}$ Department of Pathology, Vocational Technical School of Nanhai, Foshan, China
}

Received August 26, 2020

Revised November 16, 2020

Accepted November 19, 2020

Handling Editor: Jeong-II Choi

Correspondence

Yi Zhong

Key Laboratory of Neuroscience, School of Basic Medical Science, Guangzhou Medical University, Xinzao, Pangyu

District, Guangzhou 511436, China

Tel: +86-20-37103244

Fax: $+86-20-37103244$

E-mail: victoria0720@126.com

*These authors contributed equally to this work.

\begin{abstract}
Background: Lumbar disc herniation (LDH) is a common cause of radicular pain, but the mechanism is not clear. In this study, we investigated the engagement of toll-like receptor 4 (TLR4) and the nuclear factor-kappa B (NF-אB) in radicular pain and its possible mechanisms.

Methods: An LDH model was induced by autologous nucleus pulposus (NP) implantation, which was obtained from coccygeal vertebra, then relocated in the lumbar $4 / 5$ spinal nerve roots of rats. Mechanical and thermal pain behaviors were assessed by using von Frey filaments and hotplate test respectively. The protein level of TLR4 and phosphorylated-p65 ( $p-p 65$ ) was evaluated by western blotting analysis and immunofluorescence staining. Spinal microglia activation was evaluated by immunofluorescence staining of specific relevant markers. The expression of proand anti-inflammatory cytokines in the spinal dorsal horn was measured by enzyme linked immunosorbent assay.

Results: Spinal expression of TLR4 and $\mathrm{p}-\mathrm{NF}-\mathrm{K} B$ ( $\mathrm{p}-\mathrm{p} 65)$ was significantly increased after NP implantation, lasting up to 14 days. TLR4 was mainly expressed in spinal microglia, but not astrocytes or neurons. TLR4 antagonist TAK242 decreased spinal expression of $\mathrm{p}-\mathrm{p} 65$. TAK242 or NF-kB inhibitor pyrrolidinedithiocarbamic acid alleviated mechanical and thermal pain behaviors, inhibited spinal microglia activation, moderated spinal inflammatory response manifested by decreasing interleukin (IL)$1 \beta$, IL-6, tumor necrosis factor- $\alpha$ expression and increasing IL-10 expression in the spinal dorsal horn.

Conclusions: The study revealed that TLR4/NF-KB pathway participated in radicular pain by encouraging spinal microglia activation and inflammatory response.
\end{abstract}

Key Words: Cytokines; Hyperalgesia; Intervertebral Disc; Microglia; Neuralgia; NFkappa B; Nucleus Pulposus; Pain; Toll-Like Receptor 4.

\section{INTRODUCTION}

Lumbar disc herniation (LDH) is a common cause of radic- ular pain, typically manifested as hyperalgesia, allodynia, and sometimes spontaneous pain [1]. It compromises patients' life quality and work capability, and thus becomes (c) This is an open-access article distributed under the terms of the Creative Commons Attribution Non-Commercial License (http://creativecommons.org/licenses/by-nc/4.0/), which permits unrestricted non-commercial use, distribution, and reproduction in any medium, provided the original work is properly cited.

(c) The Korean Pain Society, 2021
Author contributions: Lirong Zhu: Investigation; Yangliang Huang: Project administration; Yuming Hu: Investigation; Qian Tang: Investigation; Yi Zhong: Funding acquisition. 
a socioeconomic problem that needs immediate attention [1]. Currently, the mechanism of radicular pain is still obscure.

Toll-like receptor 4 (TLR4), which is involved in innate immune response, is a trans-membrane protein with both extracellular leucine-rich repeat domains and a cytoplasmic signaling domain $[2,3]$. When binding with an endogenous or exogenous ligand, TLR4 may induce proinflammatory cytokines released by activating the nuclear factor-kappa B (NF- $\kappa$ B) or p38 pathway $[4,5]$. Previous studies found that TLR4 antagonist may relieve hyperalgesia induced by nerve injury, chemotherapy drugs or diabetes [6-8] and a genetic defect of TLR4 or its accessory factor CD14 may inhibit activation of glia cells and inflammatory pain [9-11]. However, the role and mechanism of TLR4 in radicular pain from $\mathrm{LDH}$ is not clear.

The present study was aimed at confirming the role of the TLR4/NF- $\kappa \mathrm{B}$ pathway on radicular pain, and exploring the mechanism of spinal microglia activation and subsequent inflammatory response.

\section{MATERIALS AND METHODS}

\section{Animals}

Sprague-Dawley rats (200-250 g, male) were provided by Guangdong Laboratory Animal Center. Rats were housed in separate cages under a 12-hour light/12-hour dark cycle with free access to food and water. The room temperature and humidity were constant. All animal experimental procedures were carried out in accordance with the guidelines of the International Association for the Study of Pain [12] and were approved by the Animal Care and Use Committee of Guangzhou Medical University (GD2019-143). All efforts were made to minimize animal number and their suffering.

\section{Drug administration}

TLR4 antagonist ethyl (6R)-6-[N-(2-chloro-4-fluorophenyl) sulfamoyl]-cyclohex-1-ene-1-carboxylate (TAK242, cat no. HY-11109; MedChemExpress, South Brunswick, NJ), which can effectively pass through the blood-brain barrier, was freshly prepared in saline with $5 \%$ dimethyl sulfoxide and $5 \%$ Tween 80, administered intraperitoneally 1 hour before surgery, and then once daily for 5 days with concentration based on previous studies [13].

For intrathecal administration of drugs, rats were implanted with intrathecal catheters 2 days before surgery according to previously reports [14]. Under anesthesia, a sterile polyethylene-10 (PE-10; Becton, Dickinson and
Company, Franklin Lakes, NJ) tube filled with saline was inserted through the L5-L6 intervertebral space upwards until reaching the lumbar enlargement. Any rats with hind limb paresis or paralysis after surgery were eliminated. NF- $\kappa \mathrm{B}$ inhibitor pyrrolidinedithiocarbamic acid (PDTC, $15 \mathrm{ng}$ in $10 \mu \mathrm{L}, \mathrm{P}-8765$; Sigma-Aldrich, St. Louis, MO) or vehicle was carefully injected from the distal inlet of the catheters for 5 consecutive days, beginning from 1 hour before surgery with the concentration based on previous studies $[15,16]$. Following the drug, sterile saline $(7 \mu \mathrm{L})$ was given to ensure the drug reaching the subarachnoid space.

\section{LDH model}

LDH was modeled by autologous nucleus pulposus (NP) implantation described by previous reports $[17,18]$. The transverse processes were exposed by dissecting the paraspinous muscles from the spinous processes. Hemilaminectomy was performed in the left L4-L5 segment and the lumbar nerve roots were exposed by carefully removing the facet joint. NP (about $10 \mathrm{mg}$ ) was collected from the coccygeal intervertebral discs which were exposed between two vertebral bodies ventrally, and was instantly relocated on the recently exposed nerve roots without any compression. For the sham group, rats experienced the same harvesting step, but the NPs were not implanted.

\section{Pain behavioral tests}

Mechanical pain thresholds were measured by employing a set of Von Frey filaments $(0.41,0.70,1.20,2.04,3.63$, $5.50,8.51$, and $15.14 \mathrm{~g}$ ) with an up-down method described previously [19]. The first applied stimulus was $2.04 \mathrm{~g}$, if there was no paw withdrawal, the next stronger stimulus was given. If the paw was withdrawn, a weaker stimulus was given. Stimuli were administered to the surface of the hindpaws, lasting 6-8 seconds. Brisk withdrawal or licking of the paw was regarded as a positive response.

Thermal pain thresholds were assessed by a plantar test (cat no 7370; Ugo Basile, Gemonio, Italy) [20]. The plantar surface of the rat's foot was placed on a glass floor over a radiant heat source. Three values of paw withdrawal latency (PWL) were measured for each animal in each test course. The hindpaws were tested alternately with at least 5 minutes intervals between sequential tests. The three values of PWL per animal were averaged as the result of the test.

Baselines of mechanical and thermal thresholds were assessed repetitively 3 times (day -2 , day -1 , and day 0 ) before surgery. Those performing the behavioral tests were blinded to the groups of rats. 


\section{Western blotting analysis}

The L4-L5 spinal cords were harvested and flooded with liquid nitrogen immediately, then the ipsilateral dorsal horn quadrants were separated and homogenized in Tris buffer ( $15 \mathrm{mmol} / \mathrm{L}, \mathrm{pH} 7.6)$ containing protease inhibitor cocktail (1:100, cat no. AR1183; Boster Biological Technology, Wuhan, China) and phosphatase inhibitor (1:100, cat no. AR1182; Boster Biological Technology). After homogenization and sonication on ice, the samples were centrifuged at $14,000 \times \mathrm{g}$ for 20 minutes at a low temperature. The supernatant was extracted and stored at $-80^{\circ} \mathrm{C}$ before Western blotting.

The total protein concentration of samples was calculated by bicinchoninic acid protein assay. Proteins were separated by gel electrophoresis (sodium dodecyl sulfatepolyacrylamide gel electrophoresis) and then transferred onto a polyvinylidene fluoride membrane (cat no. 1620264; Bio-Rad Laboratories, Inc., Hercules, CA). After blocking for 1 hour at room temperature, the membranes were incubated with TLR4 (mouse, 1:1,000, cat no. sc-293072; Santa Cruz Biotechnology, Inc., Dallas, TX) or $\beta$-actin (rabbit, 1:1,000, cat no. 4967; Cell Signaling Technology, Danvers, MA), phosphorylated-p65 (p-p65, rabbit, 1:1,000; cat no. 3033; Cell Signaling Technology), or p65 (rabbit, 1:1,000, cat no. 8242; Cell Signaling Technology) antibody overnight at $4^{\circ} \mathrm{C}$. After being washed in phosphate-buffered saline (PBS) repeatedly, the membranes were incubated with goat anti-mouse or goat anti-rabbit horseradish peroxidase-conjugated immunoglobulin G (IgG) (1:5,000, cat no. ab205719 or ab205718; Abcam, Cambridge, UK) for 1 hour at room temperature, then were washed again, as above. The immune complex was detected by ECL kit (cat no. 35055; Pierce Biotechnology, Rockford, IL). The band intensities were quantified by densitometry with a computer-assisted imaging analysis system (IBAS 2.0; Kontron, Augsburg, Germany).

\section{Immunofluorescence staining}

Rats were sequentially perfused with saline and cold $4 \%$ paraformaldehyde (PFA, cat no. 158127; Sigma-Aldrich) in phosphate buffer (0.1 M, pH 7.4). The L4-L5 spinal cords were taken out, post-fixed with $4 \%$ PFA solution for 1-3 hours and then dehydrated in $30 \%$ sucrose solution for 2 days at $4^{\circ} \mathrm{C}$. The spinal cords were sliced into sections transversely with $25 \mu \mathrm{m}$ thickness by a cryostat $\left(-20^{\circ} \mathrm{C}\right.$, CM1900; Leica Biosystems, Wetzlar, Germany) and processed for immunofluorescence staining.

Spinal sections were blocked with $3 \%$ donkey serum in 0.3\% Triton X-100 (X100; Sigma-Aldrich) for 0.5-1 hours at room temperature and incubated with TLR4 (rabbit 1:400, cat no. AF7017; Affinity Bioscience Pty Ltd, Scoresby, Australia) or ionized calcium binding adaptor molecule-1 (Iba1, mouse 1:500, cat no. sc32725; Santa Cruz Biotechnology, Inc., Dallas, TX) antibody overnight at $4^{\circ} \mathrm{C}$, followed by incubation with goat anti-rabbit $\mathrm{Cy}^{3}$-conjugated IgG (1:400, cat no. BA1032; Boster Biological Technology) or goat antimouse fluoresceine isothiocyanate (FITC)-conjugated IgG (cat no. BA1101; Boster Biological Technology) for 1 hour at room temperature. For double immunofluorescence staining, primary antibodies for TLR4 (1:400) were incubated together with anti-neuronal specific nuclear protein (NeuN, neuronal marker, mouse 1:500, cat no. ab104224; Abcam) or anti-glial fibrillary acidic protein (GFAP, astrocyte marker, mouse 1:500, cat no. sc33673; Santa Cruz Biotechnology, Inc.) or anti-Iba-1 (microglia marker, mouse 1:500, cat no. sc32725; Santa Cruz Biotechnology, Inc.) respectively, followed by a mixture of goat anti-mouse FITC- and goat anti-rabbit $\mathrm{Cy}^{3}$-conjugated IgG (1:400). The stained sections were examined with a fluorescence microscope (Leica Biosystems), and images were captured with a charge coupled device (CCD) spot camera (Leica Biosystems). To avoid error, the main parameters such as gain value and exposure time in image acquisition were standardized across sessions.

\section{Enzyme linked immunosorbent assay (ELISA)}

The dorsal quadrants of the L4-L5 spinal dorsal horn were rapidly harvested and homogenized in PBS followed by centrifugation at $4^{\circ} \mathrm{C}$ for $15 \mathrm{~min}$ at $14,000 \times \mathrm{g}$. The supernatants were collected to detect the concentrations of TNF- $\alpha$, IL-1 $\beta$, IL-6, and IL-10 using corresponding ELISA kits (cat no. EK0526, EK0393, EK0412, EK0418; Boster Biological Technology). According to the manufacturer's instructions, the absorbance was detected at $450 \mathrm{~nm}$ (A450) and a standard curve was delineated based on the absorbance of standards.

\section{Statistical analysis}

All data reported were means \pm standard error of means, and were analyzed with SPSS 13.0 (SPSS Inc., Chicago, IL). Western blotting, immunofluorescence and ELISA data were analyzed by one-way analysis of variance followed by Tukey's post hoc analysis. Data of behavioral tests were analyzed with the unpaired Student's $t$-test. The criterion of statistical significance was $P<0.05$. 

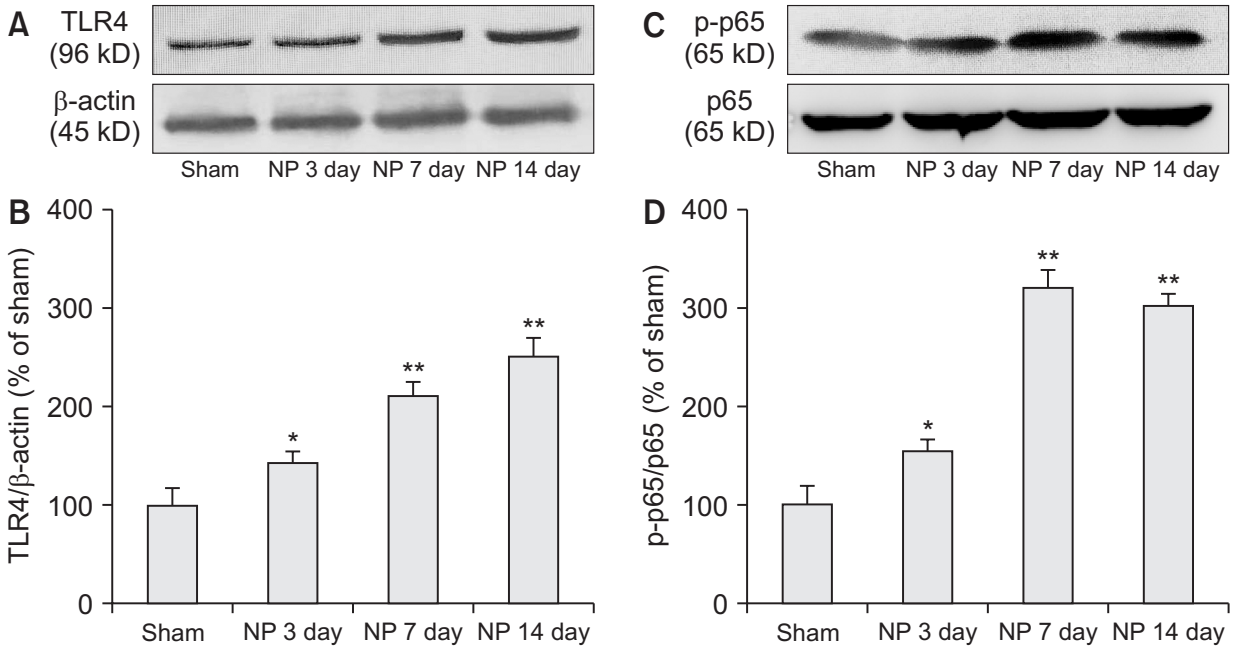

Fig. 1. The protein level of $(A, B)$ toll-like receptor 4 (TLR4) and (C, D) phosphorylated-p65 (p-p65) in spinal dorsal horn of rats with nucleus pulposus (NP) implantation $(* P=0.002$ or $0.001, * * P<0.001$, $n=5$ /group). Error bars indicate standard error of means.

\section{RESULTS}

\section{Spinal TLR4 and p-p65 expression was increased after NP implantation}

Western blotting analysis found spinal TLR4 and p-p65 expression was significantly increased from day $3 \mathrm{NP}$ implantation and lasted up to day 14 (Fig. 1A, B: ${ }^{*} P=0.002$, ${ }^{* *} P<0.001$, Fig. 1C, D: $\left.{ }^{*} P=0.001,{ }^{* *} P<0.001\right)$. Immunofluorescence staining also confirmed the up-regulation of TLR4 expression $\left({ }^{*} P=0.001,{ }^{* *} P<0.001\right.$; Fig. 2E-I). More interestingly, the up-regulation of TLR4 mainly appeared in the ipsilateral spine (Fig. 2A-C). Negative control sections, in which only the secondary antibody but not the primary antibody was incubated, were used for testing the antibody specificity (Fig. 2D). Double immunofluorescence staining showed that the TLR4 primarily coexisted with microglia (Fig. 2J-L), but not with astrocytes (Fig. 2M-0) or neurons (Fig. 2P-R).

\section{TLR4 antagonist and NF- $\kappa$ B inhibitor alleviated pain behaviors of rats with NP}

The role of TLR4 and NF- $\kappa \mathrm{B}$ in pain behaviors were tested by successive delivery of TLR4 antagonist TAK242 (intraperitoneal [i.p.], $3 \mathrm{mg} / \mathrm{kg}$ ) or NF- $\mathrm{KB}$ inhibitor PDTC (intrathecal [i.t.], $15 \mathrm{ng}$ in $10 \mu \mathrm{L}$ ), beginning from 1 hour before implantation and once daily for 5 days. NP implantation significantly decreased the mechanical PWT and thermal PWL of the ipsilateral, but not contralateral, hindpaws $\left({ }^{*} P\right.$ $=0.001,{ }^{* *} P<0.001$; Fig. 3A, B) which indicating that NP implantation induced unilateral mechanical allodynia and thermal hyperalgesia. Both TAK242 and PDTC increased the PWT and PWL of rats with NP implantation (vs. vehicle, ${ }^{\#} P=0.002$; Fig. 3C-F). Therefore, TLR4 and NF- $\kappa B$ may be involved in radicular pain from NP implantation.

\section{TLR4 antagonist TAK242 inhibited NF- $\kappa B$ activation}

TLR4 antagonist TAK242 (i.p., $3 \mathrm{mg} / \mathrm{kg}$ ) was delivered for 5 days and spinal tissues were harvested on day 7 and day 14 after surgery for western blotting analysis. TAK242 evidently weakened spinal p-p65 expression on day 7 and day 14 (**P $<0.001$; Fig. 4C, D), without changing TLR4 protein levels (Fig. 4A, B). It indicated NF- $\kappa \mathrm{B}$ may be a downstream molecule of TLR4 in the radicular pain model.

\section{TAK242 and PDTC inhibited spinal microglia activation and inflammatory response}

Spinal tissues were harvested on day 7 after surgery for assessing the effect of drugs on microglia activation and inflammatory response. Microglia-specific marker Iba1 positive areas in the NP and NP+ vehicle group were significantly increased (vs. sham, ${ }^{* *} P<0.001$; Fig. 5B, C, D, F, H); TAK242 and PDTC distinctly reduced Iba-1 positive areas (vs. vehicle, ${ }^{\# \#} P<0.001$; Fig. 5D-H). Antibody specificity was also tested (Fig. 5A). Spinal expression of proand anti-inflammatory cytokines in different groups was detected by ELISA kits. IL-1 $\beta$, IL-6, and TNF- $\alpha$ expression was decreased by TAK242 and PDTC $\left({ }^{* *} P<0.001\right.$; Fig. 6A$\mathrm{C}$ ), and both drugs increased anti-inflammatory cytokine IL-10 expression ${ }^{* *} P<0.001$; Fig. 6D). The data indicated that TLR4/NF- $\kappa \mathrm{B}$ activation may promote spinal microglia activation and inflammatory response.

\section{DISCUSSION}

In the present study, LDH was induced by autologous NP implantation. Spinal expression of TLR4 and p-p65 was significantly increased after NP implantation, lasting up 

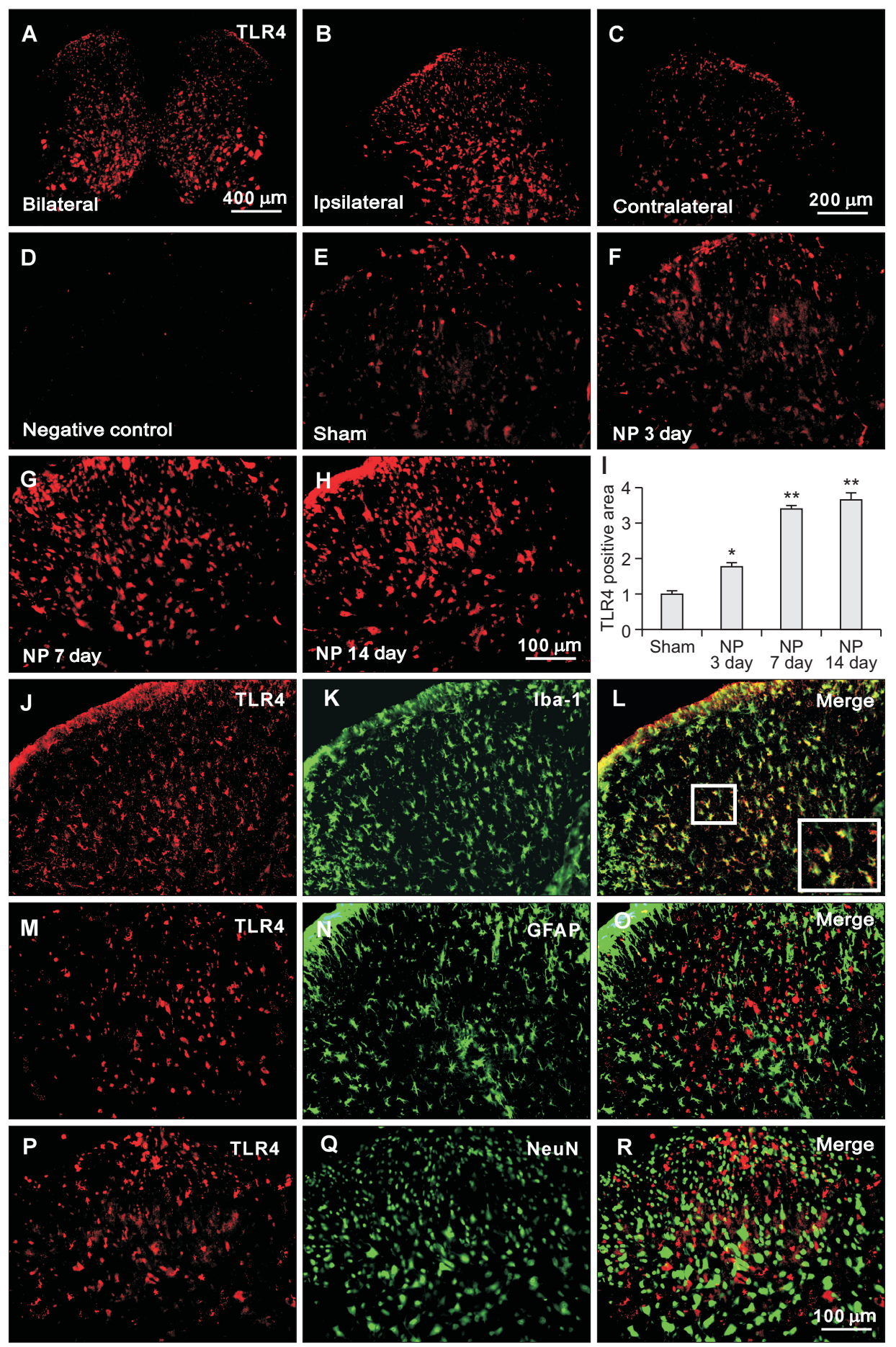

Fig. 2. (A-C) Expression of toll-like receptor 4 (TLR4) in bilateral spinal dorsal horn of rats with nucleus pulposus (NP). (D) Specificity of antibody is tested by negative control $(n=2)$. $(E-I)$ Expression of TLR4 in ipsilateral spinal dorsal horn of different groups $(* P=0.001, * * P<$ $0.001, n=4$ /group). (J-R) Double immunofluorescence staining of TLR4 (red) with cellular markers (green): ionized calcium binding adaptor molecule-1 (Iba1, microglia marker), glial fibrillary acidic protein (GFAP, astrocyte marker) and neuronal specific nuclear protein (NeuN, neuronal marker) respectively. $(\mathrm{H})$ The histogram shows lba-1 positive area of spinal dorsal horn in different groups. Scale bar $(A)=400 \mu \mathrm{m}$, Scale bars $(B, C)=200$ $\mu \mathrm{m}$, Scale bars $(\mathrm{D}-\mathrm{H}, \mathrm{J}-\mathrm{R})=100 \mu \mathrm{m}$. Error bars indicate standard error of means.

to 14 days. TLR4 was mainly expressed in spinal microglia, but not in astrocytes or neurons. Successive delivery of TLR4 antagonist TAK242 decreased spinal p-p65 expression. TAK242 or NF-кB inhibitor PDTC alleviated pain behaviors and inhibited both spinal microglia activation and spinal inflammatory response. The results demonstrated that the TLR4/NF-кB pathway may be involved in radicular pain through promoting spinal microglia activation and inflammatory response.
Typical symptoms of radicular pain from LDH are unilateral long-lasting low back pain and pain radiating into the leg and foot $[21,22]$. Initially, it was believed that herniated discs may mechanically compress the nerve roots or spinal cord, which was the source of pain [23]. But this poorly explained the fact that the degree of compression to the nerve roots was not positively correlated with the severity of pain sensation for some patients [23], thus other mechanisms other than compression may be involved in 

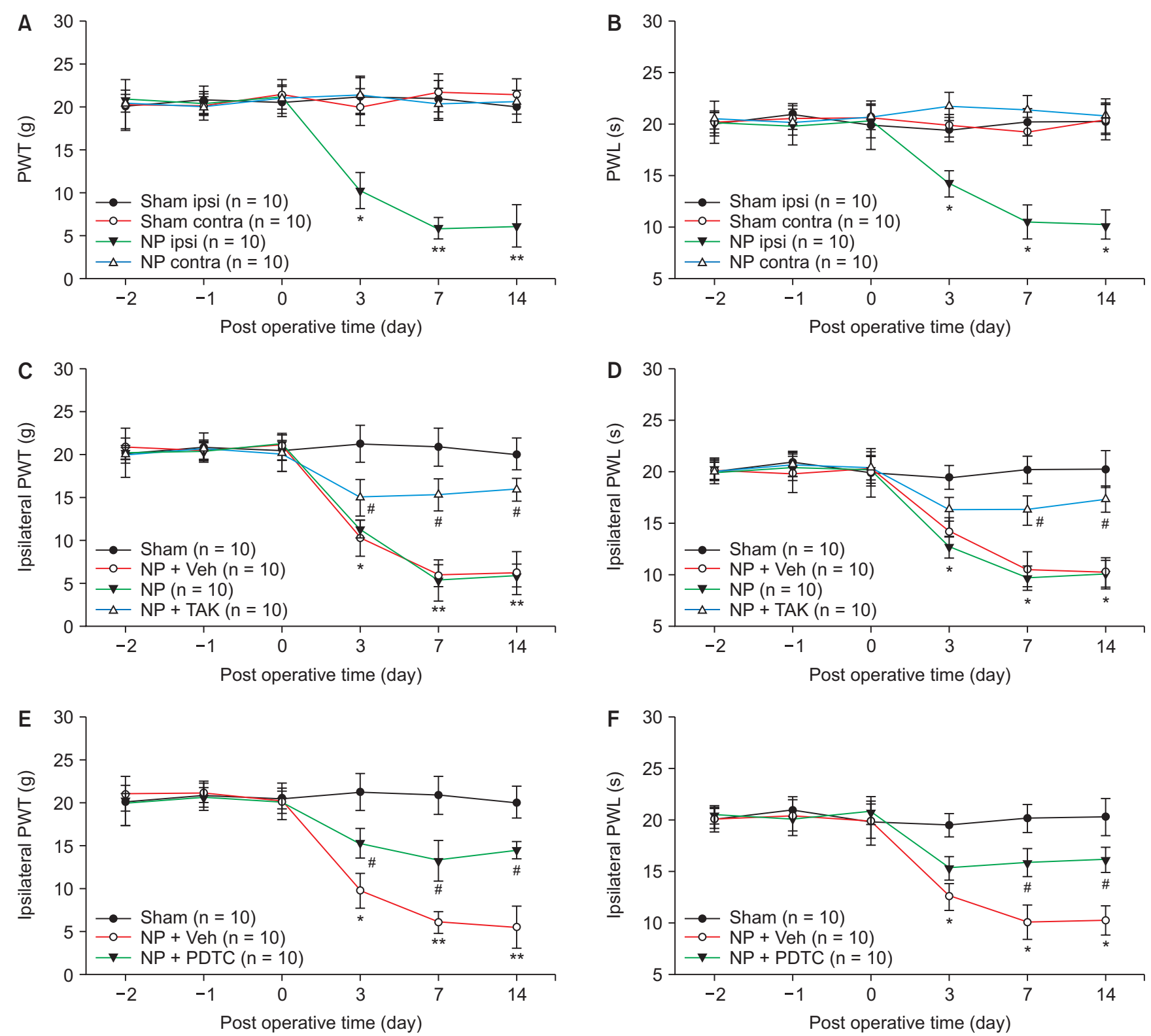

Fig. 3. (A, B) Nucleus pulposus (NP) implantation decreased mechanical and thermal thresholds in ipsilateral but not contralateral hindpaws. Toll-like receptor 4 antagonist TAK242 (TAK) (C, D) and nuclear factor-kappa B inhibitor pyrrolidinedithiocarbamic acid (PDTC) (E, F) alleviate mechanical and thermal pain behaviors of rats with NP (vs. sham, ${ }^{*} P=0.001,{ }^{*} * P<0.001$; vs. NP + vehicle [Veh], ${ }^{\#} P=0.002 ; \mathrm{n}=10$ /group). Error bars indicate standard error of means. PWT: paw withdrawal threshold, PWL: paw withdrawal latency, ipsi: ipsilateral hindpaws, contra: contralateral hindpaws.

radicular pain. Data from our reports as well as our peers' reports revealed that levels of spinal pro-inflammatory cytokines and neuroinflammation marker $18 \mathrm{kDa}$ translocator protein were increased after $\mathrm{LDH}$, which demonstrated that neuroinflammation response may be a potential reason of radicular pain $[16,24,25]$. However, the mechanism of neuroinflammation needs to be further investigated.

TLR4 is a transmembrane protein that is predominantly expressed in microglia of the central nervous system $[26,27]$. Involvement of TLR4 initiates intracellular signaling pathways, leading to the synthesis and secretion of inflammatory cytokines and chemokines, typically by activating NF- $\kappa \mathrm{B}$ and subsequent gene transcription and protein synthesis $[4,28]$. TLR4 mediated inflammatory response is involved in multiple neurodegenerative diseases such as Parkinson's disease, Alzheimer disease, and depression [29-32]. The role of TLR4 in neuropathic pain has attracted more attention in recent years. It has been reported that TLR4 antagonist may relieve hyperalgesia induced by nerve injury, chemotherapy drugs, or diabetes [6-8], and a genetic defect of TLR4 or its accessory factor CD14 may inhibit glia cells activation, as well as moderate inflammatory pain [9-11]. In the present study, the role and mechanism of TLR4 on radicular pain is first reported 

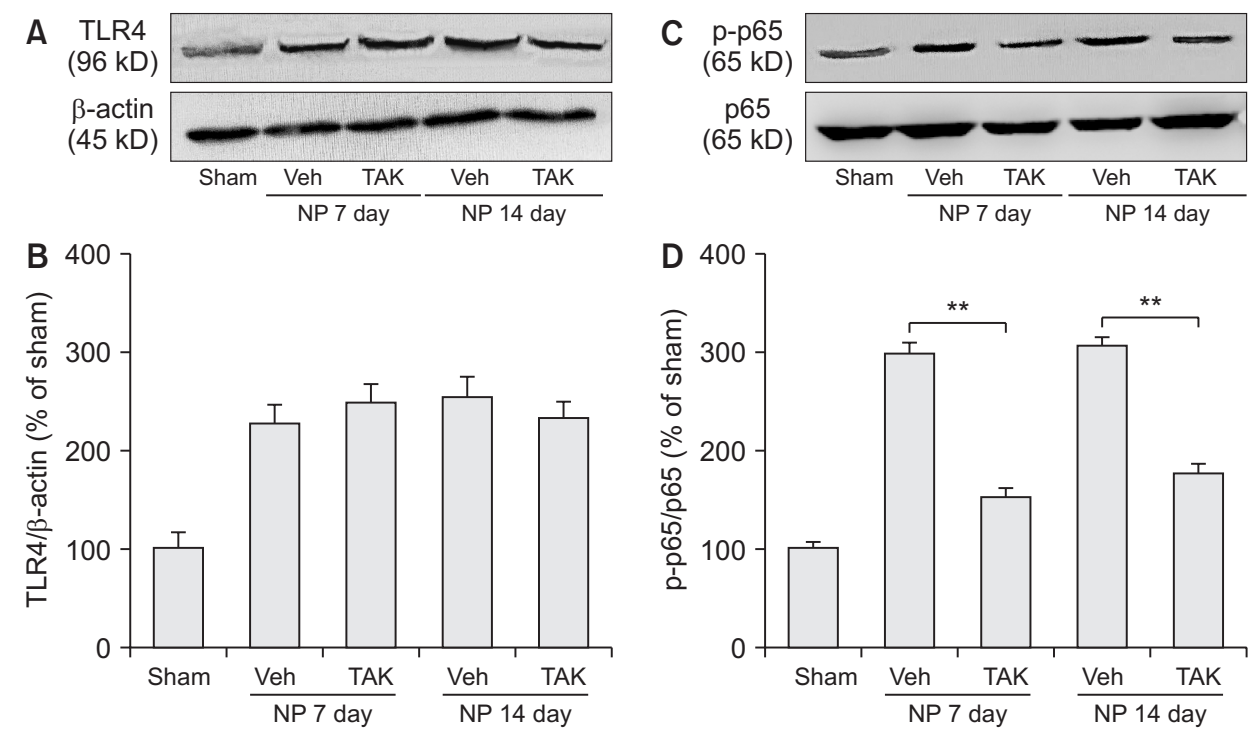

Fig. 4. TAK242 (TAK) decreases spinal phosphorylated-p65 (p-p65) expression (C, D) without changing toll-like receptor 4 (TLR4) expression (A, B). Error bars indicate standard error of means. NP: nucleus pulposus, Veh: vehicle. ${ }^{*} * P<0.001 ; \mathrm{n}=$ 5/group.
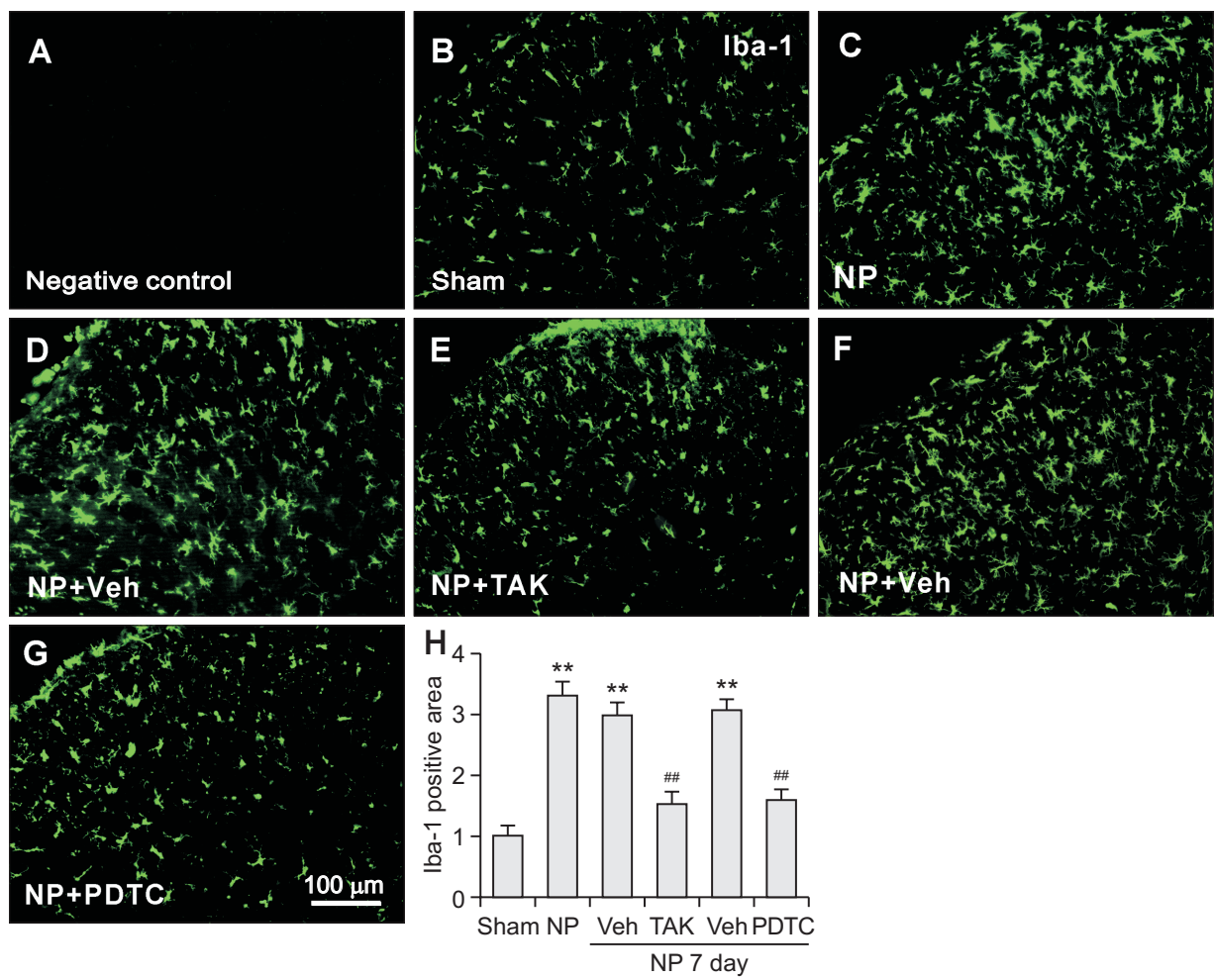

Fig. 5. TAK242 (TAK) or pyrrolidinedithiocarbamic acid (PDTC) reduces microglia specific marker ionized calcium binding adapter molecule-1 (Iba-1) expression in rats with nucleus pulposus (NP) (vs. sham, ${ }^{*} * P<0.001$; vs. NP + vehicle [veh], ${ }^{\# \#} P<0.001 ; n=4$ /group). Scale bars (A$\mathrm{G})=100 \mu \mathrm{m}$. (H) The histogram shows the Iba-1 positive area of spinal dorsal horn in different groups. Error bars indicate standard error of means. in a rat model of LDH. We find that TLR4 and its downstream molecule $\mathrm{NF}-\kappa \mathrm{B}$ are upregulated in the ipsilateral spinal dorsal horn of rats with NP implantation (Figs. 1, 2). Successively delivery of TLR4 antagonist or NF- $\kappa$ B inhibitor for 5 days significantly elevate mechanical and thermal pain thresholds that are reduced by NP implantation, and the analgesia effect lasted up to 14 days (Fig. 3). These results clearly confirm the engagement of the TLR4/NF- $\mathrm{B}$ pathway in radicular pain. Interestingly, western blotting analysis finds that intraperitoneal delivery of TLR4 an- tagonist TAK242 significantly reduces p-p65 expression, but doesn't change the TLR4 level itself (Fig. 4). As proved by other scientists, TAK242, with high permeability to the blood brain barrier, has evident analgesia effect after both intraperitoneal and intrathecal administration [33,34]. The present result also reveals that TAK242 acts as an antagonist that may block downstream signal of TLR4, but not an inhibitor which inhibits or hydrolyzes TLR4 itself. And this viewpoint is consistent with other reports which revealed that TAK242 decreased TLR4 downstream mol- 

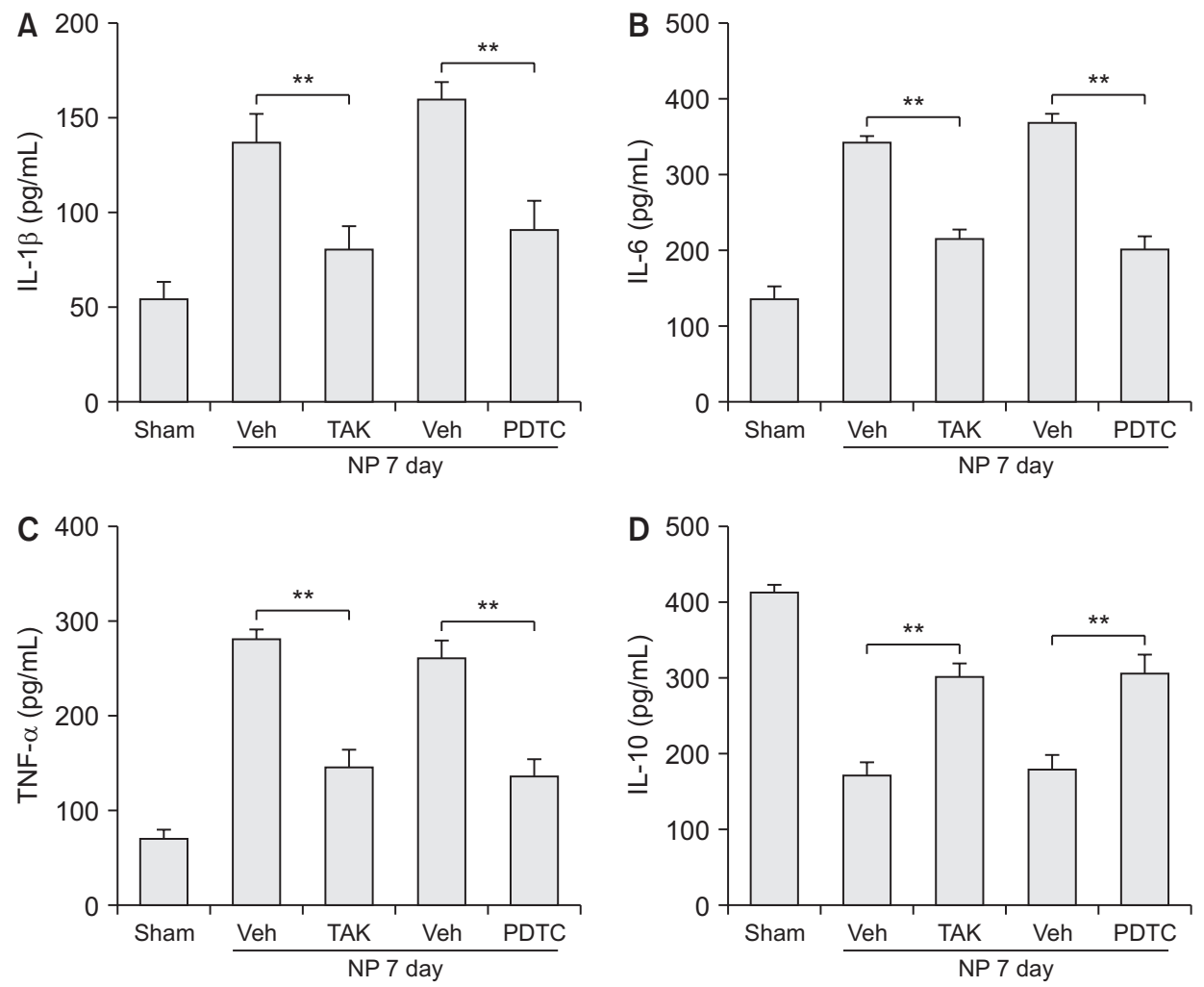

Fig. 6. Both TAK242 (TAK) and pyrrolidinedithiocarbamic acid (PDTC) reduce spinal expression of interleukin (IL)-1 $1 \beta$, IL6 , and tumor necrosis factor- $\alpha$ (TNF- $\alpha$ ), increase IL-10 expression of rats with nucleus pulposus (NP) $(* * P<0.001 ; \mathrm{n}=$ 4 /group). Error bars indicate standard error of means. Veh: vehicle.

ecules without changed TLR4 expression in brain tissues in the rat model of migranee [34]. As TLR4 is exclusively located in microglia, we deduce that this pathway may effect microglia activation.

Microglia, acting as resident macrophages in the central nervous system, could release pro-inflammatory cytokines such as TNF- $\alpha$, IL-1 $\beta$, and IL- 6 when activated in neuropathic pain conditions [35]. Data from our team and other teams has proved microglia activation contributes to radicular pain from LDH $[17,36,37]$. However, the mechanism of microglia activation is still obscure. The morphological specifics of microglial activation were cell body hypertrophy, thickened and retracted processes, increased cell numbers, and increased staining of microglial specific markers, such as Iba-1 and CD11b [38]. Previously, we proved that spinal microglia were activated mainly in the ipsilateral spine of rats with NP implantation, paralleled with pain behaviors which always appear in the ipsilateral hindpaws [17,37]. In the present study, TAK242 or PDTC obviously alleviate pain behaviors and synchronously decrease the Iba-1 positive area in the ipsilateral spinal dorsal horn, demonstrating that the TLR4/NF- $\kappa \mathrm{B}$ pathway may be an important reason for pain development and microglia activation.

What's more, we find spinal levels of pro-inflammatory cytokines (IL-1 $\beta$, IL- 6 , and TNF- $\alpha$ ) are reduced and anti- inflammatory cytokine IL-10 expression is elevated by TAK242 and PDTC. The pro-inflammatory cytokines may directly sensitize neurons in the dorsal root ganglion or spinal dorsal horn, which exaggerates pain sensation [16,39-41]. On the contrary, IL-10 down-regulates sodium channels in dorsal root ganglion neurons [42]. Clinical studies found that the serum levels of TNF- $\alpha$, IL-6, and IL-8 in patients with radicular pain were significantly higher [43-45], and anti-inflammatory cytokine IL-10, but not IL-4, was lower than that of healthy subjects [43]. In an animal model of LDH, expression of IL- $1 \beta$, TNF- $\alpha$, and IL-6 in the spinal dorsal horn or dorsal root ganglia was increased $[17,46]$. The mechanical allodynia was attenuated by inhibition of pro-inflammatory cytokines or using the neutralizing antibody in rats with NP implantation or nerve root compression [47-49]. These reports suggested the imbalance between anti- and pro-inflammatory cytokines may be a crucial reason for radicular pain and restoring balance from upstream molecules may the benefit of relieving radicular pain. Together with the present study, we may deduce that targeting spinal microglia activation and inflammatory response by inhibiting upstream signals such as TLR4 may benefit radicular pain relief.

In summary, our study demonstrates that the spinal TLR4/NF- $\kappa B$ pathway is involved in radicular pain by activating microglia and promoting inflammatory response. 


\section{CONFLICT OF INTEREST}

No potential conflict of interest relevant to this article was reported.

\section{FUNDING}

This work was supported by grants from the Characteristic Innovation Project of Ordinary University of Guangdong Province (No. 2019KTSCX145), Guangdong Basic and Applied Basic Research Foundation (No. 2020A1515010131), National Natural Science Foundation of China (No. 81600968).

\section{ORCID}

Lirong Zhu, https://orcid.org/0000-0001-6163-5497

Yangliang Huang, https://orcid.org/0000-0001-7088-5033

Yuming $\mathrm{Hu}$, https://orcid.org/0000-0002-0516-0295

Qian Tang , https://orcid.org/0000-0002-3029-5255

Yi Zhong, https://orcid.org/0000-0002-8115-4356

\section{REFERENCES}

1. Waddell G. Low back pain: a twentieth century health care enigma. Spine (Phila Pa 1976) 1996; 21: 2820-5.

2. Peri F, Piazza M. Therapeutic targeting of innate immunity with Toll-like receptor 4 (TLR4) antagonists. Biotechnol Adv 2012; 30: 251-60.

3. Li C, Yan Y, Cheng J, Xiao G, Gu J, Zhang L, et al. Toll-like receptor 4 deficiency causes reduced exploratory behavior in mice under approach-avoidance conflict. Neurosci Bull 2016; 32: 127-36.

4. Oh SH, Lee HY, Ki YJ, Kim SH, Lim KJ, Jung KT. Gabexate mesilate ameliorates the neuropathic pain in a rat model by inhibition of proinflammatory cytokines and nitric oxide pathway via suppression of nuclear factor- $\kappa$ B. Korean J Pain 2020; 33: 30-9.

5. Huang L, Chen C, Zhang X, Li X, Chen Z, Yang C, et al. Neuroprotective effect of curcumin against cerebral ischemiareperfusion via mediating autophagy and inflammation. J Mol Neurosci 2018; 64: 129-39.

6. Xing F, Zhang W, Wen J, Bai L, Gu H, Li Z, et al. TLR4/NF- $\kappa$ B signaling activation in plantar tissue and dorsal root ganglion involves in the development of postoperative pain. Mol Pain 2018; 14: 1744806918807050.

7. Zhang H, Li Y, de Carvalho-Barbosa M, Kavelaars A, Heijnen CJ, Albrecht PJ, et al. Dorsal root ganglion infiltration by macrophages contributes to paclitaxel chemotherapy- induced peripheral neuropathy. J Pain 2016; 17: 775-86.

8. Liu P, Yuan HB, Zhao S, Liu FF, Jiang YQ, Guo YX, et al. Activation of $\mathrm{GABA}_{\mathrm{B}}$ receptor suppresses diabetic neuropathic pain through toll-like receptor 4 signaling pathway in the spinal dorsal horn. Mediators Inflamm 2018; 2018: 6016272.

9. Tanga FY, Nutile-McMenemy N, DeLeo JA. The CNS role of Toll-like receptor 4 in innate neuroimmunity and painful neuropathy. Proc Natl Acad Sci U S A 2005; 102: 5856-61.

10. Cao L, Tanga FY, Deleo JA. The contributing role of CD14 in toll-like receptor 4 dependent neuropathic pain. Neuroscience 2009; 158: 896-903.

11. Buchanan MM, Hutchinson M, Watkins LR, Yin H. Toll-like receptor 4 in CNS pathologies. J Neurochem 2010; 114: 13-27.

12. Zimmermann M. Ethical guidelines for investigations of experimental pain in conscious animals. Pain 1983; 16: 109-10.

13. Moser VA, Uchoa MF, Pike CJ. TLR4 inhibitor TAK-242 attenuates the adverse neural effects of diet-induced obesity. J Neuroinflammation 2018; 15: 306.

14. Yang J, Xie MX, Hu L, Wang XF, Mai JZ, Li YY, et al. Upregulation of N-type calcium channels in the soma of uninjured dorsal root ganglion neurons contributes to neuropathic pain by increasing neuronal excitability following peripheral nerve injury. Brain Behav Immun 2018; 71: 52-65.

15. Zang Y, He XH, Xin WJ, Pang RP, Wei XH, Zhou LJ, et al. Inhibition of NF-kappaB prevents mechanical allodynia induced by spinal ventral root transection and suppresses the reexpression of Nav1.3 in DRG neurons in vivo and in vitro. Brain Res 2010; 1363: 151-8.

16. Huang Y, Zang Y, Zhou L, Gui W, Liu X, Zhong Y. The role of TNF-alpha/NF-kappa B pathway on the up-regulation of voltage-gated sodium channel Nav1.7 in DRG neurons of rats with diabetic neuropathy. Neurochem Int 2014; 75: 112-9.

17. Huang Y, Li Y, Zhong X, Hu Y, Liu P, Zhao Y, et al. Src-family kinases activation in spinal microglia contributes to central sensitization and chronic pain after lumbar disc herniation. Mol Pain 2017; 13: 1744806917733637.

18. Kaneuchi Y, Sekiguchi M, Kameda T, Kobayashi Y, Konno SI. Temporal and spatial changes of $\mu$-opioid receptors in the brain, spinal cord and dorsal root ganglion in a rat lumbar disc herniation model. Spine (Phila Pa 1976) 2019; 44: 85-95.

19. Chaplan SR, Bach FW, Pogrel JW, Chung JM, Yaksh TL. Quantitative assessment of tactile allodynia in the rat paw. J Neurosci Methods 1994; 53: 55-63.

20. Hargreaves K, Dubner R, Brown F, Flores C, Joris J. A new and sensitive method for measuring thermal nociception in cutaneous hyperalgesia. Pain 1988; 32: 77-88.

21. Valat JP, Genevay S, Marty M, Rozenberg S, Koes B. Sciatica. Best Pract Res Clin Rheumatol 2010; 24: 241-52.

22. Gadjradj PS, Arts MP, van Tulder MW, Rietdijk WJR, Peul WC, Harhangi BS. Management of symptomatic lumbar disk herniation: an international perspective. Spine (Phila Pa 1976) 2017; 42: 1826-34. 
23. Andrade P, Hoogland G, Garcia MA, Steinbusch HW, Daemen MA, Visser-Vandewalle V. Elevated IL-1 $\beta$ and IL- 6 levels in lumbar herniated discs in patients with sciatic pain. Eur Spine J 2013; 22: 714-20.

24. Zhong Y, Huang Y, Hu Y, Xu M, Zhu L, Deng Z. SFKs/p38 pathway is involved in radicular pain by promoting spinal expression of pro-inflammatory cytokines in a rat model of lumbar disc herniation. Spine (Phila Pa 1976) 2019; 44: E111221.

25. Albrecht DS, Ahmed SU, Kettner NW, Borra RJH, CohenAdad J, Deng H, et al. Neuroinflammation of the spinal cord and nerve roots in chronic radicular pain patients. Pain 2018; 159: 968-77.

26. Yousefi N, Sotoodehnejadnematalahi F, Heshmati-Fakhr N, Sayyah M, Hoseini M, Ghassemi S, et al. Prestimulation of microglia through TLR4 pathway promotes interferon beta expression in a rat model of Alzheimer's disease. J Mol Neurosci 2019; 67: 495-503.

27. Xiang HF, Cao DH, Yang YQ, Wang HQ, Zhu LJ, Ruan BH, et al. Isoflurane protects against injury caused by deprivation of oxygen and glucose in microglia through regulation of the Toll-like receptor 4 pathway. J Mol Neurosci 2014; 54: 664-70.

28. Zusso M, Lunardi V, Franceschini D, Pagetta A, Lo R, Stifani $\mathrm{S}$, et al. Ciprofloxacin and levofloxacin attenuate microglia inflammatory response via TLR4/NF-kB pathway. J Neuroinflammation 2019; 16: 148.

29. Kouli A, Horne CB, Williams-Gray CH. Toll-like receptors and their therapeutic potential in Parkinson's disease and $\alpha$-synucleinopathies. Brain Behav Immun 2019; 81: 41-51.

30. Azam S, Jakaria M, Kim IS, Kim J, Haque ME, Choi DK. Regulation of toll-like receptor (TLR) signaling pathway by polyphenols in the treatment of age-linked neurodegenerative diseases: focus on TLR4 signaling. Front Immunol 2019; 10: 1000.

31. Balducci C, Frasca A, Zotti M, La Vitola P, Mhillaj E, Grigoli E, et al. Toll-like receptor 4-dependent glial cell activation mediates the impairment in memory establishment induced by $\beta$-amyloid oligomers in an acute mouse model of Alzheimer's disease. Brain Behav Immun 2017; 60: 188-97.

32. Wang Y, Xu J, Liu Y, Li Z, Li X. TLR4-NF- $\kappa$ B signal involved in depressive-like behaviors and cytokine expression of frontal cortex and hippocampus in stressed C57BL/6 and ob/ob mice. Neural Plast 2018; 2018: 7254016.

33. Woller SA, Ravula SB, Tucci FC, Beaton G, Corr M, Isseroff RR, et al. Systemic TAK-242 prevents intrathecal LPS evoked hyperalgesia in male, but not female mice and prevents delayed allodynia following intraplantar formalin in both male and female mice: the role of TLR4 in the evolution of a persistent pain state. Brain Behav Immun 2016; 56: 271-80.

34. Su M, Ran Y, He Z, Zhang M, Hu G, Tang W, et al. Inhibition of toll-like receptor 4 alleviates hyperalgesia induced by acute dural inflammation in experimental migraine. Mol
Pain 2018; 14: 1744806918754612.

35. Jeon YH, Youn DH. Spinal gap junction channels in neuropathic pain. Korean J Pain 2015; 28: 231-5.

36. Cho HK, Ahn SH, Kim SY, Choi MJ, Hwang SJ, Cho YW. Changes in the expressions of Ibal and calcitonin generelated peptide in adjacent lumbar spinal segments after lumbar disc herniation in a rat model. J Korean Med Sci 2015; 30: 1902-10.

37. Zhong Y, Huang YL, Hu YM, Zhu LR, Zhao YS. Puerarin alleviate radicular pain from lumbar disc herniation by inhibiting ERK-dependent spinal microglia activation. Neuropeptides 2018; 72: 30-7.

38. Saghaei E, Abbaszadeh F, Naseri K, Ghorbanpoor S, Afhami M, Haeri A, et al. Estradiol attenuates spinal cord injury-induced pain by suppressing microglial activation in thalamic VPL nuclei of rats. Neurosci Res 2013; 75: 316-23.

39. Gruber-Schoffnegger D, Drdla-Schutting R, Hönigsperger C, Wunderbaldinger G, Gassner M, Sandkühler J. Induction of thermal hyperalgesia and synaptic long-term potentiation in the spinal cord lamina I by TNF- $\alpha$ and IL- $1 \beta$ is mediated by glial cells. J Neurosci 2013; 33: 6540-51.

40. Chirila AM, Brown TE, Bishop RA, Bellono NW, Pucci FG, Kauer JA. Long-term potentiation of glycinergic synapses triggered by interleukin $1 \beta$. Proc Natl Acad Sci U S A 2014; 111: 8263-8.

41. Zhong Y, Zhou LJ, Ren WJ, Xin WJ, Li YY, Zhang T, et al. The direction of synaptic plasticity mediated by $\mathrm{C}$-fibers in spinal dorsal horn is decided by Src-family kinases in microglia: the role of tumor necrosis factor-alpha. Brain Behav Immun 2010; 24: 874-80.

42. Shen KF, Zhu HQ, Wei XH, Wang J, Li YY, Pang RP, et al. Interleukin-10 down-regulates voltage gated sodium channels in rat dorsal root ganglion neurons. Exp Neurol 2013; 247: 466-75.

43. Wang K, Bao JP, Yang S, Hong X, Liu L, Xie XH, et al. A cohort study comparing the serum levels of pro- or anti-inflammatory cytokines in patients with lumbar radicular pain and healthy subjects. Eur Spine J 2016; 25: 1428-34.

44. Kraychete DC, Sakata RK, Issy AM, Bacellar O, SantosJesus R, Carvalho EM. Serum cytokine levels in patients with chronic low back pain due to herniated disc: analytical cross-sectional study. Sao Paulo Med J 2010; 128: 259-62.

45. Pedersen LM, Schistad E, Jacobsen LM, Røe C, Gjerstad J. Serum levels of the pro-inflammatory interleukins 6 (IL-6) and -8 (IL-8) in patients with lumbar radicular pain due to disc herniation: a 12-month prospective study. Brain Behav Immun 2015; 46: 132-6.

46. Liu ZH, Miao GS, Wang JN, Yang CX, Fu ZJ, Sun T. Resolvin D1 inhibits mechanical hypersensitivity in sciatica by modulating the expression of nuclear factor $-\kappa \mathrm{B}$, phospho-extracellular signal-regulated kinase, and pro- and antiinflammatory cytokines in the spinal cord and dorsal root ganglion. 
Anesthesiology 2016; 124: 934-44.

47. Sasaki N, Kikuchi S, Konno S, Sekiguchi M, Watanabe K. Anti-TNF-alpha antibody reduces pain-behavioral changes induced by epidural application of nucleus pulposus in a rat model depending on the timing of administration. Spine (Phila Pa 1976) 2007; 32: 413-6.

48. Olmarker K, Nutu M, Størkson R. Changes in spontaneous behavior in rats exposed to experimental disc herniation are blocked by selective TNF-alpha inhibition. Spine (Phila $\mathrm{Pa}$ 1976) 2003; 28: 1635-41.

49. Rothman SM, Winkelstein BA. Cytokine antagonism reduces pain and modulates spinal astrocytic reactivity after cervical nerve root compression. Ann Biomed Eng 2010; 38: 2563-76. 